\title{
LODOVICO DOLCE NELLA STORIA DELLE IDEE FEMMINISTE
}

\section{LODOVICO DOLCE IN THE HISTORY OF FEMINIST IDEAS}

\author{
Ada Boubara \\ Aristotle University of Thessaloniki, Thessaloniki, Greece \\ boubara@itl.auth.gr
}

Recibido: octubre de 2021

Aceptado: noviembre de 2021

Parole chiave: Lodovico Dolce, comportamento, donne, maritate.

Key words: Lodovico Dolce, behavior, women, married.

Riassunto: Lodovico Dolce (1508/1510-1568), umanista e studioso del Cinquecento, fu tra gli intellettuali che parteciparono alla Querelle des Femmes durante il periodo rinascimentale. Proprio in questo ambito si colloca il suo trattato Dialogo di M. Lodovico Dolce della institution delle donne secondo li tre stati che cadono nella vita humana. L'obiettivo dell'articolo consiste nell'esaminare ed evidenziare le idee di Dolce sulla "institution della maritata" esposte nel secondo libro del Dialogo, presentare il profilo femminile proposto e le virtù di cui deve essere dotata una donna coniugata nella società del XVI secolo.

\begin{abstract}
Lodovico Dolce (1508/ 1510-1568), a humanist and scholar of the $16^{\text {th }}$ century, was among the intellectuals involved in the Querelle des Femmes, during the Renaissance period. His treatise Dialogo di M. Lodovico Dolce della institution delle donne secondo li tre stati, che cadono nella vita humana represents precisely this context. The purpose of this article is, on one hand, to examine and highlight Dolce's argumentation on the "institution della maritata" set out in the second book of the Dialogo; on the other hand, its aim is to present the suggested female profile and the virtues that a married woman should have in the XVIth century society.
\end{abstract}

\section{Introduzione}

La tematica della Querelle des Femmes e le testimonianze scritte relative a questo argomento sono molte e come emerge dallo studio di Virginia Cox, Women's Writing in Italy, 1400-1650 (Cox, 2008) e di Mercedes Arriaga su "Le scrittrici marchigiane:

(Questa ricerca è risultato del Progetto «Men for Women. Voces Masculinas en la Querella de las Mujeres» (PID2019-104004GB-I00), Ministerio de Economía y Competitividad).

Revista Internacional de Pensamiento Político - I Época - Vol. 16 - 2021 - [149-160] - ISSN 1885-589X 
un giallo letterario" (Arriaga Flórez, 2008) possiamo notare che nell'ambito italiano, già nel tredicesimo e nel quattordicesimo secolo, sono presenti testi in cui appaiono versi scritti da donna, come ad esempio quelli di Giustina Levi Perotti, Elisabetta Trebbiani, Monna Nina e altre che con le loro opere poetiche offrono un contributo all'avvio della Querelle (Arriaga Flórez et al, 2012). Inoltre, come sostiene Daniele Cerrato nel suo articolo Sorelle di QuereIle. Poetesse dell'al-Andalus, trobairitz e poetesse italiane del Duecento e Trecento (Cerrato, 2015):

il dibattito/polemica legato ai meriti e alla dignità delle donne, trova ampio spazio durante i secoli XV e XVI, in una serie di trattati composti da Mario Equicola, Galeazzo Capra, Torquato Tasso, ai quali si affiancano i testi di autrici quali Isotta Nogarola, Arcangela Tarabotti, Moderata Fonte, Cassandra Fedele, Lucrezia Marinella. Si tratta di un confronto letterario che si sviluppa soprattutto attraverso la prosa, ma vi prenderanno parte anche poetesse tra cui Laura Terracina, Laura Battiferri, Veronica Gambara e Veronica Franco (Cerrato, 2015: 232).

I testi medievali del XIII secolo di Umberto da Romans, Gilberto da Tournai e Stefano di Borbone presentano la posizione della donna entro un certo stato sociale e vengono definiti i "loro ruoli fondamentali: sposate, vedove, vergini e fanciulle, monache e religiose. Ne esce un quadro ora conturbante, ora grottesco ed esilarante: alle donne, di qualunque condizione sociale siano, è sempre proposto di non fare qualcosa" (Casagrande, 1978: retro di copertina). Inoltre, come si vede anche dall'articolo "Sposa, figlia, sorella e vecchia matre». Invecchiare donna in età moderna, tra demografia e cultura di P. Renée Baernstein (Baernstein, 2006) nello spazio europeo: una donna [...] misurava i passaggi della propria vita in base alle differenti relazioni intrattenute con gli uomini nel corso del tempo: era una vergine sotto il tetto del padre, diventava poi una moglie quando era "menata" dal marito. Poi, di solito, veniva la maternità e, se la donna sopravviveva ai rigori delle gravidanze e dei parti, diventava una vedova dopo la morte del coniuge. Di fronte alla legge e alla sua famiglia, erano queste le condizioni che definivano una donna; le tappe più importanti, mentre cresceva, erano quelle in cui usciva o entrava nella casa di un uomo (Baernstein, 2006: 213).

Nel periodo dell'Umanesimo e nel successivo Rinascimento, le riflessioni intorno alla questione femminile (Fahy,1956; Sanson \& Lucioli, 2016) e alla posizione della donna, diventano sempre più frequenti.

In particolare, come si nota dall'articolo di Paola Cosentino Tragiche eroine. Virtù femminili fra poesia drammatica e trattati sul comportamento (Cosentino, 2006):

All'inizio del XVI secolo, la condizione femminile diventa infatti oggetto di una intensa riflessione che tocca i più diversi ambiti e penetra in tutti i generi letterari. Tanto nella novella quanto nella lirica cortigiana, tanto nei trattati quanto nei poemi cavallereschi la donna è al centro di una rinnovata curiosità. Come testimonia il progressivo affermarsi di una vera e propria «querelle des femmes», non soltanto i compiti, ma anche la stessa natura femminile vengono ora interamente rimessi in discussione (Cosentino, 2006: 69).

Entro allora tale dibattito intorno alla superiorità 0 inferiorità delle donne, affiorano dei "trattati di institutio della donna, generalmente orientati a fornire norme e regole che definiscono una vera e propria grammatica del comportamento" (Cosentino, 2006: 69). 
In questo quadro si inserisce Lodovico Dolce $^{1}$ (Cicogna, 1862; Dionisotti, 1970; Romei, 1991; Terpening, 1997) il quale partecipò al dibattito sulla condizione della donna nella società del sedicesimo secolo. II suo contributo alla Querelle des Femmes fu il Dialogo di M. Lodovico Dolce della institution delle donne secondo li tre stati, che cadono nella vita humana? una dissertazione di carattere pedagogico di importante valore culturale. Un trattato che "al suo interno avviene infatti il processo di sostituzione e scambio dell'archetipo aristocratico con l'archetipo borghese" (Sberlati, 1997:162).

II Dialogo di Lodovico Dolce, della institution delle donne secondo li tre stati, che cadono nella vita humana "modellato sul trattatello del Vives"3 (Cosentino, 2006: 82) De institutione foeminae Christianae (Del Nero, 2006), si svolge tra gli interlocutori Flaminio e Dorotea. È composto da tre libri, ognuno dei quali presenta i ragionamenti e le regole di comportamento per le tre fasi della vita delle donne (Matthews

1. Lodovico Dolce (Venezia 1508/1510-Venezia 1568) fu umanista e studioso veneziano del XVI secolo, scrittore prolifico che ha collaborato a lungo con il tipografo Gabriele Giolito de'Ferrari. Ebbe rapporti con molti celebri intellettuali e artisti della sua epoca, tra cui Pietro Aretino, Tiziano, Pietro Bembo e altri. Scrisse trattati, commedie, tragedie, testi in versi e in prosa. Inoltre, fu abile traduttore di testi classici e curatore di opere di grandi scrittori.

2. Prima edizione a Venezia per i tipi di Gabriele Giolito de' Ferrari nel 1545.

3. Juan Luis Vives (Valencia 1492-Bruges 1540) fu spagnolo umanista e studioso. La sua opera De institutione foeminae Christianae, stampata per prima volta nel 1524 ma dopo ripubblicata e rivista nel 1538, è un trattato importante del periodo rinascimentale da cui emergono le regole che deve seguire una donna nella società del suo tempo e ovviamente secondo i dettami della fede cristiana.
Grieco \& Brevaglieri, 2001; Cox, 1992), "vergine in attesa di matrimonio, [...] sposa e madre; casta vedova devota alla memoria del marito" (Sberlati, 1997: 124).

Così il tema del matrimonio "è stato trattato durante il secolo da molti eruditi e fu argomento di testi vari, laici e religiosi, in versi e in prosa" (Sanson, 2015: 50) e l'obiettivo di questo testo consiste nel presentare una disamina delle idee di Lodovico Dolce sulla "institution della maritata" esposte nel secondo libro del Dialogo; raffigurare il ritratto femminile proposto e le virtù della donna coniugata nella società del sedicesimo secolo in quanto:

anche se i dilatati orizzonti mentali del Rinascimento avevano consentito l'emergere e il diffondersi di una nuova idea di femminilità, [...] scopriamo in realtà che la condizione storica della donna, [...] era socialmente ancora irreggimentata nel tradizionale ordine tripartito degli 'stati' verginale, maritale e vedovile. II suo ruolo non aveva altre dimensioni all'infuori di queste tre: vergine in attesa di matrimonio, di frequente con un uomo impostole da legami di interesse dinastico o finanziario; sposa e madre; casta vedova devota alla memoria del marito (Sberlati, 1997:124).

\section{I ragionamenti di Lodovico Dolce sulla "institution della maritata"}

Per seguire il filo del discorso dello scrittore veneziano dedicato all'istituzione della donna maritata, è indispensabile ritornare al primo libro del trattato in cui si presenta il percorso formativo della vergine (Boubara, 2020) e le virtù di cui deve essere dotata allo scopo di essere pronta e giustamente istruita per arrivare alla soglia 
delle nozze. Quindi per quanto riguarda l'educazione della fanciulla:

notiamo che il percorso formativo inizia già dal valore dell'allattamento materno. Passa in seguito ai giochi adatti, insiste sui buoni costumi e la virtù e presenta l'importanza della religione e della gestione domestica. Nello stesso tempo, la ragazza deve essere colta e aver letto dei testi che esaltino la bellezza dell'anima. È necessario, inoltre, che la giovinetta sia timida, che segua un contegno di verginità e castità e che eviti il male dell'ozio. Similmente, ornamenti di bellezza, vestiti di lusso, giochi d'azzardo, come le carte o i dadi, sono interamente vietati. La selezione e la valutazione dello sposo, infine, è obbligo del padre e solo lui ha l'incarico e la responsabilità della scelta giusta (Boubara, 2020: 58).

Le considerazioni esposte delineano il ritratto immaginario della giovinetta vergine, condizione necessaria per il percorso della futura sposa, preparata per inserirsi nella seconda fase della sua vita, quella di donna maritata. Di conseguenza, la sposa, per poter concepire il suo ruolo entro questo nuovo contesto, ha bisogno della formazione appropriata e il secondo libro del Dialogo 4 si occupa di questo tema.

Osservando gli argomenti trattati constatiamo che Dolce in qualità di educatore attraverso la voce dell'interlocutore Flaminio, inizia il suo discorso elogiando la bellezza del giardino, lo spazio scelto per il dialogo con Dorotea, e subito coglie l'occasione per paragonare l'incanto di questo posto florido con la grazia del matrimonio, poiché "il matrimonio, quando è formato da quegli ordini, et conservato da quelle conditioni che si convengono a cosa di tanta importanza, esso è appunto simile a un

4. Tutte le citazioni sono riportate dal testo originale dell'edizione del 1545 per i tipi di Gabriele Giolito de' Ferrari.
Giardino ripieno di tutte le contentezze e felicità humane" (Dolce, 1545: 39).

Lo scrittore partendo da questa premessa generale per lo sposalizio, continua il suo ragionamento introduttivo e sottolinea "che avanti, che la [...] figliuola entri in casa del novello marito, è mestiero, che si riduca molto bene nella memoria l'ufficio e il fine del matrimonio, per poter poi sodisfar pienamente con l'opera a quello, che ella havrà prima considerato con l'intelletto" (ibídem) ed espone il principio che "I'Huomo e la Donna altro non essere che una carne istessa. Onde congiunti insieme, di due corpi divengono uno solo; e ne formano quel mirabile Hermaphrodito, che non si può dividere" (ibídem). Di conseguenza "è adunque l'ufficio del matrimonio congiungere il Marito e la moglie con sì stretta unione, che non siano più che uno; e il fine è generare" (Dolce, 1545: 40).

Lodovico Dolce per consolidare il suo ragionamento presenta l'opinione di Francesco Barbaro secondo il quale "il matrimonio essere perpetuo congiungimento di huomo e di donna, ordinato per cagione di crear legitimamente figliuoli, e di fuggire adulterio" (ibídem). Per enfatizzare la sua tesi, il trattatista fornisce degli esempi dall'antichità, e in particolare fa riferimento ai Romani e ai Lacedemoni; evidenzia il modo in cui venivano affrontati coloro che non avevano prole e sottolinea che in quelle società creare figli ed eredi era un atto di importanza vitale. Successivamente, il ragionamento si sposta sulla tematica centrale dell'educazione della maritata e sulle regole da rispettare rigorosamente ed inevitabilmente.

La sposa, infatti, non deve considerare il suo passaggio al matrimonio come un momento di festa, di divertimento, bensì 
essa deve affrontare il vincolo matrimoniale come se entrasse in un "albergo casto e santo: nel quale facendo ella col marito quella perfetta unione, che dicemo, vivi felice tutto il suo tempo" (ibídem). Viene chiarito subito che questa unione ha come capo il marito e la moglie è costretta ad amarlo e onorarlo, cioè la donna deve sapere che "tutto il governo della moglie dependa dal Marito" e che "al Marito appartiene il comandare, e a lei l'ubbidire è richiesto" (Dolce, 1545: 40). Tuttavia, viene chiarito che la donna non deve affrontare il concetto dell'obbedienza come sinonimo della servitù perché, "quando servendo al Marito, è servire a se medesima, e se pure ella meritasse nome di servitù; è ripiena di tanta soavità e dolcezza, che avanza ogni libertà" (ibídem).

Di nodale importanza della riflessione di Dolce è che dal momento in cui l'unione dei coniugi è un legame sacro, la donna, proprio a causa della religiosità dell'atto dello sposalizio, deve passare il giorno delle nozze:

non in Danze e Conviti, come è costume si consumi, ma tutto si spenda in rivolger preghi a Dio, che sì come egli solo è Auttore del matrimonio; così a quello (la sua merce) porga il favore della sua gratia: senza la quale niuna operatione fra mortali hebbe mai buono succedimento (Dolce, 1545: 41).

Viene enfatizzato il valore e il peso delle nozze dal punto di vista religioso, come momento di suprema grazia concessa da Dio, bisogna affrontarlo con il massimo rispetto e la necessaria spiritualità, ed inoltre, divertimenti e svaghi non sono compatibili con il vero significato del giorno delle nozze. Nel successivo ragionamento Dolce situa la giovane donna maritata nell'ambiente della dimora dello sposo ed inizia la presentazione di tutte le virtù che la coniuge deve possedere da quel momento in poi. In questo contesto, è fondamentale che "fra tutte le virtù pertinenti alla maritata, ne n'habbia due, che dell'altre tengano la maggioranza: [...] queste sono castità in lei, et amore verso il marito" (ibídem).

La virtù della castità è fondamentale per l'educazione della donna e questo è un ragionamento evidenziato e commentato da Lodovico Dolce al primo libro del Dialogo dedicato all'istituzione della vergine. Anche per la donna maritata la castità è una delle sue doti più preziose, un pregio necessario e importante che:

dee portar seco dalla casa del padre: [...] la moglie, che offende questa, offende primieramente Dio, con l'autorità di cui è fatto il matrimonio; ed a cui giurò la purità del letto matrimoniale. Da poi fa ingiuria al marito, [..] fa ingiuria alla charità del prossimo; perché non se ne trova a lei alcuno maggiore di colui, che le è padre, fratello, compagno, marito e Signore. Diparte la unione, [..] Spezza il legame santissimo del congiungimento humano, rompe la fede, [...] Leva la compagnia civile: offende le leggi e la patria: flagella il padre, la madre, le sorelle, i fratelli, i parenti, e gli amici. È di cattivo esempio ai suoi: infama la famiglia: e poi, ch'è divenuta madre, è madre così iniqua e scelerata, che i figliuoli udir ragionar di lei non possono senza vergogna; né ricordare il nome del padre senza dubbio d'esser suoi figliuoli (Dolce, 1545: 41).

Dopo questo lungo elenco dei mali provenienti dall'assenza della castità, lo scrittore afferma che per le donne maritate la verginità appartiene alla fede e al marito, così se la maritata non rispetta i dettami della buona condotta in virtù della castità e la offre ad altri "commette il peccato del latrocinio" (Dolce, 1545: 42). Nella riflessione che segue, il discorso si concentra 
sulla tematica della corporatura. La donna non ha diritti sulla gestione del suo corpo il quale "è tutto in poter del marito" (Dolce, 1545: 43).

In seguito, viene affrontato il tema dell'adulterio, il quale corrompe il matrimonio, provocando anche danni ai figli ma anche al valore dello sposalizio dato che "Due altri beni ne corrompe l'Adulterio, per cagion de quali il matrimonio è formato: I'uno è la prole; l'altro la facultà: perciò che la prole, come io dico, fa incerta: ed è cagione, che si consumano le facultà" (ibídem). Viene presentato un lungo elenco di casi di donne che per mantenere la loro castità hanno preferito la morte.

Successivamente il trattatista espone la riflessione relativa all'amore della moglie nei confronti del marito che è una delle due virtù fondamentali della maritata messa in evidenza nell'incipit del suo discorso. Per questa considerazione è importante notare che lo scrittore sostenga che le donne coniugate, debbano amare il marito come amano sé stesse e come amano i propri familiari cioè "lui essere a lei in luogo di padre, di madre, di fratello, e di sorella" (Dolce, 1545: 44). II discorso, inoltre, raffigura il rapporto matrimoniale come un legame che supera l'amicizia perché "vince di gran lunga tutte le altre amicitie" (ibídem) e in più la coppia ha un'anima e un corpo. Da questo accoppiamento "un solo huomo si forma: atteso, che l'animo della moglie dee vivere in quello del marito; et ella a lui, come a parte migliore di se medesima, in tutte le cose ubbedire, et render honore" (ibídem).

Lo scrittore, per rafforzare il suo pensiero e chiarire in maniera indiscutibile la posizione della donna nel matrimonio, dichiara che essa, essendo più debole dell'uomo, ha bisogno di protezione, "Iontana dal ma- rito, riman sola, ignuda, et soggetta a tutte le offese" (Dolce, 1545: 44). Solo vicino al consorte, solo se è accompagnata dal marito, suo signore, "dove ella si trova, ivi è la sua patria, la sua casa, i suoi parenti, i suoi domestici, et tutte le sue ricchezze" (ibídem).

L'interlocutore Flaminio, nel corso della sua argomentazione, fornisce degli esempi di donne maritate valorose che non hanno tradito i principi della fede, dell'amore e della castità e hanno onorato la sacra unione delle nozze come Issicratea moglie di Mitridate Re di Ponto, Flacilla Nonio Prisco, Sulpitia, la moglie di Ferdinando Gonzaga Conte di CasteIla, Alceste, Laodamia, Paolina moglie di Seneca, Giulia figliuola di Giulio Cesare, Cornelia l'ultima moglie di Pompeo e tante altre. L'elenco dei nomi enumerati è lungo e copre un arco di tempo dall'antichità fino ai giorni del trattatista, il quale esalta e descrive il comportamento di tutte queste donne maritate e in più presenta un caso di particolare interesse come dimostrazione di assoluta devozione al marito. Si tratta di una giovane moglie "chiamata Gamma: bella di corpo, ma molto più di animo" (Dolce, 1545: 46) la quale per non violare le leggi matrimoniali, per non essere infedele al marito e per vendicare la morte del consorte, finge di essere felice futura sposa dell'uomo che ha ucciso suo marito. Proprio nel momento della celebrazione delle nozze si uccidono tutti e due bevendo del vino avvelenato. Gamma lascia il suo ultimo sospiro esclamando:

hora me n'andrò contenta a trovare il mio Sinato. Da poi rivoltasi a Sinorige, seguitò. Et tu crudele e scelerato huomo puoi ordinare ai tuoi servi, che invece delle pompe delle nozze, che malamente hai desiderate, apparecchino sepoltura al tuo corpo (Dolce, 1545: 46). 
II trattatista nel corso della presentazione di questa sua riflessione sottolinea che la donna maritata ha sempre bisogno di mettere la vita del consorte al di sopra di tutte le sue comodità e unisce questo aspetto al sentimento dell'amore. Per evidenziare il valore basilare dell'amore nel legame matrimoniale lo scrittore dichiara che la moglie deve "amar sinceramente e con tutto l'animo il suo consorte" Dolce, 1545: 47) e sottolinea che l'amore per il marito è il primo e basilare obbligo della sposa. Chiarisce le caratteristiche di questo amore e spiega che:

conviene, che con questo amore sia congiunta una somma riverentia, e una obedienza più, che perfetta: con ciò sia cosa, che non solamente le leggi humane, ma le Divine comandano, che la moglie sia soggetta al marito: e non solamente le leggi, ma la natura istessa questa feminile soggettione dimostra (Dolce, 1545: 47).

Di grande importanza è questo punto del Dialogo poiché l'interlocutore Flaminioil trattatista Lodovico Dolce sostengono l'opinione riguardante la natura del sesso femminile. Nel matrimonio la donna deve rispettare il marito come capo e deve essere governata da lui dato che "alla Donna è così di mestiero il governo dell'huomo; come è a ciascun cielo, lo effetto di chi lo muove" (ibídem).

Per chiarire il ragionamento dell'amore e dell'ubbidienza della maritata nei confronti del suo capo-marito, lo scrittore parte dalla premessa che la donna può essere amata dallo sposo se gli è del tutto devota, se tutto quello che fa, tutto quello che pensa mira ad accontentare lui, se "tutte le sue opre corrispondino al voler di lui" (ibídem). La giovane sposa necessita essere preparata ad affrontare la vita coniugale con tutti gli aspetti positivi e negativi, il che significa i momenti belli o brutti, la ricchezza o la povertà, la salute o la malattia, la bellezza esteriore della giovinezza o la senilità poiché "il vivere humano è come il giuoco d'i Dadi" (Dolce, 1545: 48). Di conseguenza la donna maritata deve essere preparata per tutte le eventuali possibilità e deve sempre essere partecipe del bene e del male del consorte. Nello stesso contesto dei consigli per la vita coniugale viene evidenziato anche il ragionamento secondo il quale la donna necessita prestare "insieme opera non solo di Moglie, ma di Medica, e di cuoca, e (senza alcuna vergogna havere) di servente" (ibídem). L'elenco dei consigli alla giovane maritata continua, è necessario affrontare il carattere del marito, se "sarà superbo, bisogna che contra questa superbia opponga lo scudo della humiltà: se sdegnoso, quello della patietia" (Dolce, 1545: 49). In più, il trattatista afferma che la sposa non deve mai prendere in considerazione la felicità di altre maritate che magari sembrano più felici proprio perché "chi può saper quello, che giace ascoso sotto i tetti di tante case?" (ibídem).

Dunque, la donna coniugata per ottenere la felicità e la tranquillità in casa deve sapere quando è il momento giusto per parlare, per rilevare i suoi errori commessi e nello stesso tempo deve sapere quando bisogna tacere:

et se peraventura (quel, ch'è più difficili a supportare) egli trasportato da ira, o da qualche infirmità d'animo, s'inducesse a batterla; tenga alhora d'esser dalle mani di Dio per castigo de suoi peccati battuta: Anchora che di rado avverrà, che la buona moglie e prudente sia battuta dal marito (Dolce, 1545: 50).

Ciò nonostante, tutto quello che avviene nell'ambito domestico, le sofferenze, 
i disagi, le difficoltà "si debbono tener rinchiusi e sepelliti dentro le case" (Dolce, 1545: 50) per proteggere la fama e il nome del marito. In aggiunta, la maritata, "si dimostri verso di lui, quale si sogliono dimostrar le buone madri verso sì fatti figliuoli" (ibídem). In merito a tale argomento, e secondo i suggerimenti proposti, il trattatista riassume il suo ragionamento affermando che:

ora, quale egli si sia, è marito, capo, e Signore della femina: dato a lei da Dio, dalla chiesa, e dai parenti. di tanto numero d'huomini questa è la sorte et la parte sua: bisogna che si contenti, e supporti con buono animo quello, che mutare non si può: bisogna, che lo ami, che l'honori, e che l'osservi: se non per lui (che per lui, in quanto è marito, si dee amare, honorare, e osservare) per coloro, che glie l'hanno dato, e per la fede da lei obligata nel maritare (Dolce, 1545: 50).

In seguito, il discorso mette in risalto il fatto che la maritata è obbligata ad avere come assoluta priorità i bisogni del marito, e deve mirare sempre alla soddisfazione delle sue esigenze poiché in tal modo la casa sarà regnata dalla concordia, dalla pace e dalla carità. In caso contrario la loro dimora "che dovrebbe esser casa di pace e d'amore, è fatta albergo d'odio e di discordia" (Dolce, 1545: 51).

È necessario evidenziare che lo scrittore insiste sul valore fondamentale della concordia come elemento indispensabile per la felicità e la tranquillità degli sposi nel matrimonio, al contrario la discordia genera soltanto ostacoli e sventure. Per consolidare la sua riflessione il trattatista cita Pitagora e i principali insegnamenti ai suoi allievi, uno dei quali era "dalla casa la discordia" (ibídem); quindi la pace nel matrimonio si mantiene se la donna agisce costantemente nel quadro della conformità, del consenso, della comprensio- ne. In tale contesto Dolce, sotto la voce di Flaminio, afferma "che volendo nel matrimonio la pace, vi sia la concordia: la quale in buona parte è posta in mano alla moglie" (Dolce, 1545: 52); ciò significa che la maritata deve seguire il volere del marito e il suo umore, "non gli si voglia anteporre in alcuna cosa' [...] le ricchezze, le povertà; le allegrezze, gli affanni; i beni e i mali tenga communi egualmente" (Dolce, 1545: 53). Per il concetto della comunanza si rifà all'esempio dei Romani "che tra il marito e la moglie alcuna cosa si potesse dir propria né distinta" (ibídem) e al pensiero di Platone secondo cui "in una bene ordinata Republica si debba levar via queste voci Tuo e Mio" (ibídem). Così, indipendentemente dal discorso relativo al concetto della comunanza, la supremazia del marito è indiscutibile, è padrone e Signore e la donna anche se porta da casa sua dote e ricchezza considerevole, una volta maritata tutto passa al marito, mentre la donna con "la honestà, la castità, la bontà, la virtù, la obedientia, la diligentia nel governo della famiglia, e sì fatti thesori: dei quali s'ella è abondevole, è riccamente dotata d'ogni bene." (ibídem).

Quindi l'interlocutore, esaltando il valore della concordia come sfondo innegabile per un matrimonio riuscito e mettendo in evidenza le virtù preziose, riferite sopra, come doti inestimabili della donna maritata, conclude questa sua argomentazione con l'esaltazione della devozione, del rispetto e della fedeltà della sposa, il che significa che "la sua obedientia deve essere appoggiata sopra l'amore; dove quella delle fanti procede solamente dall'obligo et dalla timidità" (Dolce, 1545: 54).

II ragionamento successivo riguarda il con cui la maritata "si debba trattenere col marito nelle dimore famigliari" (ibídem). 
II trattatista presenta a tal proposito i suoi suggerimenti e le regole che la sposa deve rispettare e seguire nell'ambito del focolare domestico. Quindi "tra il marito e la moglie non doveva haver luogo ira né amaritudine alcuna. [...] che con l'amore si conviene accompagnare una grata piacevolezza e soavità di attioni" (ibídem). I coniugi non devono avere nessun segreto o desiderio nascosto, la moglie non deve essere curiosa, non deve mai parlare male al marito o offenderlo e ovviamenete mantenere la sua castità. Inoltre, deve scegliere vestiti che sono apprezzati e approvati dal marito e lo stesso vale per tutti i lavori di casa; insomma deve stare attenta a evitare le discordie.

Dopo la presentazione dei dettami del comportamento della maritata nell'ambiente della casa, il trattatista passa a ragionare sulla gelosia e sui grandi problemi che causa nel matrimonio. Per questa "acerbissima passione dell'animo" (Dolce, 1545: 55) che potrebbe dimorare nella mente e nella psiche di ambedue i coniugi, lo scrittore presenta le regole della condotta della sposa allo scopo di evitare la gelosia del marito e i problemi consecutivi che genera questo tormento disastroso. Oltre allora alla virtù della castità, la maritata ha l'obbligo di stare attenta a non creare mai sospetto al marito per le sue azioni; il che significa che non deve:

ammettere in casa persona alcuna, se non di consentimento del marito: non parlar d'alcun' huomo, se non parcamente: non mostrar molto desiderio di gire a feste, a visite, o dove che sia, se non di ordine del marito: non usare ornamenti, che avanzino il costume di lei: non pregar con molta istanza per altrui, et sì fatte cose: le quali tutte possono rendere odore di contaminato animo (Dolce, 1545: 55).
Inoltre, è da notare che da questo ragionamento emerge un elemento essenziale: se il doloroso sentimento della gelosia entra nell'anima della maritata, in primis deve pensare che il marito è il suo padrone, il suo Signore e inoltre le leggi della castità riguardano le donne. Di conseguenza queste due considerazioni sono i motivi per cui la maritata non ha diritto alla gelosia, e "dee la buona moglie tolerarlo con patientia" (Dolce, 1545: 56). La successiva riflessione riguarda la gestione domestica e come la maritata deve affrontare questo suo ruolo rilevante. Le regole sono bene precise:

dee adunque la moglie esser diligentissima in conservare tutto quello, che entra in casa, et fuggendo la prodigalità, non discenda però all'Avaritia; ma tenga una certa temperatezza, che non si accosti al poco, né al soverchio. Allegrisi di veder nel marito spese di honore, et opere di charità. Provegga, ch'alla famiglia niuna cosa manchi: [...] Non sia verso i famigliari aspra, né dura, ma benigna e piacevole. [...] che fosse severa senza asprezza, e diligente senza violentia: (Dolce, 1545: 57).

Lo scrittore-interlocutore, per consolidare il suo discorso riporta il parere dell'Apostolo Paolo il quale "aggiunge alla prudentia ed alla castità della Donna la cura delle cose della famiglia" (Dolce, 1545: 58). L'ultimo ragionamento riporta il pensiero aristotelico per sottolineare e riassumere in forma di epilogo i dettami da seguire per il governo del domicilio. Mette in evidenza il peso fondamentale del ruolo della maritata al trattamento "di tutte le cose che sono dentro la casa" (ibídem); il marito ha il compito di "aquistare" e la moglie quello di "conservare". In più la donna coniugata deve avere un atteggiamento di rispetto sia nei confronti del marito che nei confronti delle leggi matrimoniali imposte da Dio, allevare figli disciplinati e onesti, 
seguire una vita di indiscutibile castità ed eccellenti virtù, tutti elementi essenziali per il profilo ideale della donna maritata, ma soprattutto la coniuge deve sempre prendere in considerazione che "sì come non può esser fiamma senza luce; così è necessario, che la virtù sia accompagnata dall'honore"(Dolce, 1545: 62).

\section{La formazione della maritata - Conclusioni}

Sintetizzando dunque le idee di Lodovico Dolce, voce maschile che espone i dettami della buona condotta e le regole indispensabili per l'educazione della maritata, notiamo che:

per una donna il giorno della vita arriva presto, come sposa e novella madre. II primo grande cambiamento in una vita femminile era il passaggio dalla fanciullezza a un breve periodo di adolescenza, quando veniva detta "da maritare", in età da marito -cioè, postpuberalema non ancora sposata. Questa breve fase e il suo culminare nelle nozze [...] rappresentava l'apice rituale della vita di una donna, segnato da cerimonie e attenzione pubblica; un momento atteso -e spesso anticipato- con eccitazione e timore, sia dalla fanciulla che dalla sua famiglia (Baernstein, 2006: 217).

Nel secondo libro del Dialogo dedicato all'educazione della donna maritata prevale "un modello di mentalità, oltre che di femminilità, che sta alla base del moderno concetto di famiglia, intesa anche come cellula di un piu vasto tessuto sociale tenuto insieme da coesioni aggregative" (Sberlati, 1997: 162). Lodovico Dolce mira a istruire, a preparare la donna coniugata per affrontare lo stato maritale e i suoi consigli sono delle norme che vanno di pari passo con lo spirito e con le esigenze della sua epoca il che significa: l'ascesa della donna borghese e [...] la decadenza della donna aristocratica [...] si installa la perfetta donna schiettamente 'borghese', tutta chiesa e famiglia, il cui modello sociale dominera praticamente con indisturbata prevalenza sino all'emergere del movimento femminista nel nostro secolo (Sberlati, 1997: 162).

Ovviamente tutto il percorso formativo della donna si basa in primis sul valore della formazione della vergine la quale, dotata delle caratteristiche appropriate come la castità e la verginità, è in possesso dei requisiti indiscussi per passare allo stato della maritata in modo degno. Lodovico Dolce tra le norme e i suggerimenti che propone è fondamentale "quello che deve considerare la sposa prima, che ella entri in casa del novello marito" (Dolce, 1545: 4). II trattatista introduce dal primo libro la tematica dello sposalizio e si sofferma su due punti: il primo è il ruolo del padre nella scelta del marito a cui "si dava consiglio [...] che fossero diligenti consideratori nel maritar delle figliuole; non le ricchezze, ma gli huomini prudenti e di sano intelletto cercando" (Dolce, 1545: 36). II secondo punto è:

il parallelismo dello sposalizio con il lavoro "di buoni e accurati Agricoltori" (Dolce, 1545: car.36), i quali hanno l'obbligo di "riguardare in qual terreno spargano il seme" (Dolce, 1545: car.36). Quindi la giusta scelta del consorte è fondamentale perché la fanciulla senta "di haver trovato non un marito, quale si desiderava per lei: ma, che un'Angelo le sia mandato da cielo per sostegno e scorta de passi suoi" (Dolce, 1545: car.37) (Boubara, 2020: 58).

Dunque, tutta questa preparazione conduce alla creazione di una donna modello che quando entra nel suo nuovo ambiente domestico sarà in grado di affrontare con abilità e successo il ruolo di moglie, madre 
e padrona di casa. Dolce tramite il ritratto della sposa ideale suggerisce una specie di canone secondo il quale la donna coniugata deve affrontare e amare il marito come Signore e capo di tutto, quindi essere obbediente, fedele, casta, saggia nel parlare e nell'agire, deve fare di tutto per mantenere la concordia matrimoniale, saper gestire la passione della gelosia, educare i figli con disciplina, saper governare la casa proficuamente per tutta la famiglia e onorare sempre con le sue virtù sé stessa, il consorte e il suo focolare domestico. In sostanza il secondo libro del Dialogo di M. Lodovico Dolce della institution delle donne secondo li tre stati che cadono neIla vita humana offre un piano di formazione completo, finalizzato all'acquisizione di competenze adatte all'inserimento della donna nella società borghese del sedicesimo secolo. Ciò emerge anche dalle parole di Dorotea, interlocutrice del dialogo, che dopo aver sentito la presentazione dei suggerimenti per l'educazione della donna maritata espone il suo parere in merito e dichiara che "Voi, Signor Flaminio, havete vinta l'aspettation mia. Et questa vostra sposa è tale, che merita esservi invidiata da tutti gli huomini" (Dolce, 1545: 62).

\section{Bibliografia}

Arriaga Flórez, M. (2008). Le scrittrici marchigiane: un giallo letterario. Studi Umanistici Piceni, Sassoferrato, Istituto Internazionale di Studi Piceni, $n^{\circ}$ XXVIII, 161-168.

Arriaga Flórez, M., Cerrato, \& D., Rosal Nadales, M. (2012). Poetas italianas de los siglos XIII y XIV en la Querella de las mujeres. Sevilla, Arcibel.
Baernstein, P. R. (2006). «Sposa, figlia, sorella e vecchia matre». Invecchiare donna in età moderna, tra demografia e cultura, Storia delle donne, 2, Firenze University Press, 213-230.

Boubara, A. (2020). I ragionamenti di Lodovico Dolce sulla Institution della vergine. Revista de La Sociedad Española de Italianistas, vol. 14, 51-59.

Casagrande, C. (1978). Prediche alle donne del secolo XIII. Milano, Bompiani.

Cerrato, D. (2015). Sorelle di Querelle. Poetesse dell'al-Andalus, trobairitz e poetesse italiane del Duecento e Trecento. Raudem - Revista de Estudios de las Mujeres, vol. 3, 232-251.

Cicogna, E. A. (1862). Memoria intorno la vita e gli scritti di Messer Lodovico Dolce, letterato veneziano del secolo XVI. Memorie dell'Istituto Veneto di Scienze, Lettere e Arti, vol. 11, 93-200.

Cosentino, P. (2006). Tragiche eroine. Virtù femminili fra poesia drammatica e trattati sul comportamento. Italique, IX, 65-99.

Cox, V. (1992). The Renaissance dialogue: literary dialogue in its social and political contexts, Castiglione to Galileo. Cambridge, Cambridge University Press.

Cox, V. (2008). Women's Writing in Italy, 1400-1650. Baltimore, Johns Hopkins University Press.

Del Nero, V. (2006). Problemi aperti sul ‘De Institutione Foeminae Christianae' Di Juan Luis Vives. Bruniana \& Campanelliana, vol. 12, no. 1, Accademia Editoriale, 11-23.

Dionisotti, C. (1970). Dolce, Lodovico. In Enciclopedia dantesca, Roma, Istituto dell'Enciclopedia, 534-535.

Dolce, L. (1545). Dialogo di M. Lodovico Dolce della institution delle donne secon- 
do li tre stati, che cadono nella vita humana. Venezia, Gabriele Giolito de' Ferrari.

Fahy, C. (1956). Three early Renaissance Treatises on Women. Italian Studies, 11, 30-55.

Matthews Grieco, S. F., Brevaglieri, S. (Cur.). (2001). Monaca, moglie, serva e cortigiana: vita e immagine delle donne tra Rinascimento e Controriforma (Lunaris), Firenze, Morgana.

Romei, G. (1991). Dolce, Lodovico. In Dizionario Biografico degli Italiani, Roma, Istituto dell'Enciclopedia Italiana, 399405.

Sanson, H. (2015). Lodovico Dolce, Dialogo della instituzion delle donne secondo li tre stati che cadono nella vita umana (1545), Critical Texts, 30, Cambridge, MHRA.

Sanson, H., Lucioli, F. (2016). Conduct Literature for and about Women in Italy, 1470-1900: Describing and Prescribing Life. Paris, Classiques Garnier.

Sberlati, F. (1997). Dalla donna di palazzo alla donna di famiglia: Pedagogia e cultura femminile tra Rinascimento e Controriforma. I Tatti Studies in the Italian Renaissance, vol. 7, 119-174.

Terpening, R. H. (1997). Lodovico Dolce. Renaissance Man of Letters. Toronto, University of Toronto Press. 